\title{
LOS TIENTOS 68, 65 Y 67 DE OBRAS DE MÚSICA: ESTUDIO ANALÍTICO DE TRES OBRAS MAESTRAS DE CABEZÓN
}

\author{
THE TIENTOS 68, 65 Y 67 FROM OBRAS DE MÚSICA: \\ AN ANALYTICAL STUDY OF THREE MASTERPIECES BY CABEZÓN
}

\author{
Miguel A. Roig-Francolí \\ University of Cincinnati
}

\begin{abstract}
Resumen:
El presente estudio se centra en tres tientos que sin duda pueden contarse entre las obras maestras de Cabezón: los números 68, 65 y 67 de Obras de música. Este orden en particular nos permite viajar analíticamente desde un tiento largo y brillante con gran unidad modal, hasta un tiento corto e introspectivo que sin embargo incluye tres secciones, cada una de las cuales está en un modo distinto, pasando por un tiento de longitud mediana que demuestra ambigüedad estructural entre dos modos. El mundo tonal de Cabezón (y también de su tiempo) se encuentra representado en estos tres tientos en toda su riqueza y variedad. Abordamos tres aspectos de cada uno de estos tientos: organización formal, coherencia temática y relationes motívicas entre diversos sujetos, y organización modal. Lejos de ser aspectos aislados dentro del proceso musical, veremos que estos tres elementos están íntimamente relacionados en estas obras de Cabezón, y juntos constituyen y determinan las estructuras compositivas que precisamente definen estos tientos como obras maestras.
\end{abstract}

\section{Palabras clave:}

Cabezón, tiento, Obras de música, modalidad, estructura tonal, forma, coherencia temática, ambigüedad modal

\begin{abstract}
:
The present study focuses on three tientos which can undoubtedly be counted among Cabezón's masterpieces, numbers 68, 65, an 67 from Obras de música. This particular order allows an analytical journey from a long and brilliant tiento with great modal unity, to a short and introspective piece that includes three sections, each of which is in a different mode, by way of a mid-length tiento featuring structural ambiguity between two modes. Cabezón's tonal world (as well as his time's) is represented in these three tientos in all its richness and variety. We consider three aspects in each of these tientos: Formal organization, thematic coherence and motivic relationships among different subjects, and modal organization. Far from being three isolated features within the musical process, we will see that these three elements are closely related in these works by Cabezón, and together they constitute and determine the compositional structures that define these tientos as masterpieces.
\end{abstract}

Keywords:

Cabezón, tiento, Obras de música, modality, tonal structure, form, thematic coherence, modal ambiguity 
La publicación de una nueva edición crítica de las Obras de música de Antonio de Cabezón nos da la ocasión para volver a examinar con detenimiento la música del insigne organista burgalés ${ }^{1}$. Son numerosas las obras maestras entre las colecciones de música de Cabezón (Libro de cifra nueva y Obras de música). A menudo se piensa en sus variaciones al hablar de sus obras maestras, sobre todo si se tienen en cuenta las numerosas grabaciones que de ellas existen (en particular sus siempre populares Diferencias sobre el Canto llano del caballero, las tres colecciones sobre Guárdame las vacas, otras tres sobre la Pavana italiana, y las Diferencias sobre la Gallarda milanesa). Menos atención han recibido las obras maestras entre sus tientos (14 en el Libro de cifra nueva, y otros 12-o 14 según la edición-en las Obras de música). En diversas publicaciones he llamado la atención sobre varios de estos tientos magistrales por medio de estudios analíticos, en particular las piezas números 36 (tiento 10, modo 1$)^{2}$ y 64 (tiento 18 , modo 4$)^{3}$ del Libro de cifra nueva, y los números 60 (tiento 3, modo 1$)^{4}$ y 64 (tiento 7 , modo 4) $)^{5}$ de las Obras de música ${ }^{6}$.

Para el presente estudio, nos centraremos en otros tres tientos que sin duda pueden contarse entre las obras maestras del maestro burgalés: los números 68 (tiento 11, modo 6), 65 (tiento 8, modo 8) y 67 (tiento 10, modo 1) de Obras

1 CABEZÓN, 2010. Los tientos están incluidos en el volumen II de esta edición. Los doce tientos en la nueva edición siguen la numeración de la edición original de Hernando de Cabezón (1578), e incluyen las obras numeradas 58 a 69. En la edición de Pedrell/Anglés (CABEZÓN, 1966) los tientos llevan la misma numeración, de 58 a 69, pero además están identificados con un número para cada tiento, de 1 a 14. Así, en la edición de Pedrell/Anglés, Obras 58 lleva también la rúbrica "tiento 1"; Obras 59 es también el "tiento 2", etc. Nótese que Anglés cuenta con dos tientos más que la presente edición, porque incluye entre los tientos las obras tituladas "Fuga a cuatro voces" (tiento 13 en Anglés, número 79 en la presente edición) y "Ad Dominum cum tribularer" (tiento 14 en Anglés, número 109 en la presente edición). Ambas obras están basadas en la técnica de imitación canónica, y son propiamente "fugas" (en el sentido renacentista, sinónimo de "cánones") y no tientos. Charles Jacobs, por su parte, presenta los doce tientos de las Obras con los números 15-26 (precedidos por los catorce tientos del Libro) en el volumen II de JACOBS, 1967-86. Jacobs incluye las dos fugas de las Obras como los números 17 y 18 del tercer volúmen de su edición. Para complicar más las cosas, la edición de Kastner de los doce tientos y dos fugas de las Obras está ordenada modalmente, y por tanto los números no corresponden con ninguna de las otras ediciones (KASTNER, 1958).

2 ROIG-FRANCOLÍ, 2000.

3 ROIG-FRANCOLÍ, 1994.

4 ROIG-FRANCOLÍ, 1992.

5 ROIG-FRANCOLÍ, 2004.

6 Véase también mi estudio de dos tientos basados en tonos de Magníficat, la pieza número 32 (tiento 6, modo 4) del Libro, y la número 62 (tiento 5, modo 2) de Obras, en ROIG-FRANCOLÍ, 1998. La totalidad de los 28 tientos (incluyendo las dos "fugas") están analizados en ROIG-FRANCOLÍ, 1990. de música ${ }^{7}$. El primero de estos tientos es uno de la más brillantes y virtuosos de Cabezón, y presenta total unidad en su estructura tonal en el modo 6. El segundo se caracteriza por su unidad estilística y temática, y es un excelente ejemplo de ambiguiedad estructural entre dos modos. El tercero, por su parte, es breve y contemplativo, y presenta el caso más interesante de cambio modal entre todos los tientos de Cabezón. He escogido este orden en particular precisamente porque nos permite viajar analíticamente desde un tiento largo y brillante con gran unidad modal, hasta un tiento corto e introspectivo que sin embargo incluye tres secciones, cada una de las cuales está en un modo distinto, pasando por un tiento de longitud mediana que demuestra ambigüedad estructural entre dos modos. El mundo tonal de Cabezón (y también de su tiempo) se encuentra representado en estos tres tientos en toda su riqueza y variedad. El periplo, sin duda, merece mucho la pena.

En el estudio analítico que presentamos a continuación abordaremos tres aspectos de cada uno de estos tientos: organización formal; coherencia temática y relationes motívicas entre diversos sujetos; y organización modal, con atención a las características particulares de la modalidad de cada tiento. Lejos de ser tres aspectos aislados dentro del proceso musical, veremos que estos tres elementos (forma, cohesión temática y modalidad) están íntimamente relacionados en estas obras de Cabezón, y juntos constituyen y determinan las estructuras compositivas que precisamente definen estos tientos como obras maestras.

\section{OBRAS DE MÚSICA 68 (TIENTO 11), MODO 6}

\section{Forma}

Obras 68 es el más largo de los tientos de Cabezón y, junto con Obras 60 (tiento 3, modo 1), uno de los más brillantes y virtuosos. Comprende dos partes (especificadas por el compositor), y su forma es notablemente compleja, incluyendo un total de siete sujetos distintos. Los compases 1-67 constituyen la primera sección, que incluye la exposición del sujeto S1 (cc. 1-25, ejemplo 1a), dos variaciones contrapuntísticas sobre $\mathrm{S} 1$ (cc. 26-33 y 34-44) y un episodio sobre S1 estructurado en forma de dúos (cc. 44-67). El aspecto más notable de la segunda sección (cc. 67-101, sobre $\mathrm{S} 2$, ver ejemplo 1b), es la aproximación a la cadencia que cierra la sección por medio de un extenso circulo de quintas:

7 No he publicado con anterioridad ningún análisis de Obras 68 . He publicado análisis en inglés, por otro lado, de Obras 65 y Obras 67 en ROIG-FRANCOLÍ, 2000. 
cadencia en Sol en el c. 83; en Do en el c. 87; y cadencia final en Fa en el c. 91. La tercera sección, S3 (cc. 102-145) incluye dos sujetos: S3a (cc. 102-111) y S3b (111-123), ilustrados en el ejemplo 1c, y seguidos por un episodio contrapuntístico (123-145) que cierra con la presencia de S3b y de una retrogradación de S3a (bajo, cc. 136-140). La cuarta sección, S4 (cc. 145-182, ejemplo 1d) concluye la primera parte del tiento. Esta última sección es extendida por medio de una progresión canónica sobre S4 que crea un efecto de quasi-ostinato (tenor y bajo, cc. 149-167), acompańada por un triple y alto libres. En los compases 163-170 el tenor incluye cuatro de los sujetos presentados anteriormente en la primera parte, en forma de recapitulación final: S4 (cc. 1
63-165), S3b (166-167), S2 en inversión (168-169), y S3a (170), tal y como se ilustra en el ejemplo 1e.

La segunda parte empieza con un cambio a compás triple. Una breve sección que presenta el sujeto S5 (cc. 1-11, ejemplo 1f) está conectada a la sección S6 por medio de una inversión de S1 (tiple, cc. 11-12). A continuación de la sección S6 (cc. 13-24, ejemplo 1g), un episodio sobre S1 cierra el pasaje en compás triple (cc. 24-42). La vuelta a compás binario en el compás 42 introduce el sujeto $\mathrm{S} 7$ (ejemplo 1h), derivado del tono salmódico 6 (seculorum 6) ${ }^{8}$, seguido por una brillante y virtuosa sección final en estilo contrapuntístico altamente disminuido.

Ejemplo 1: Sujetos en Obras 68
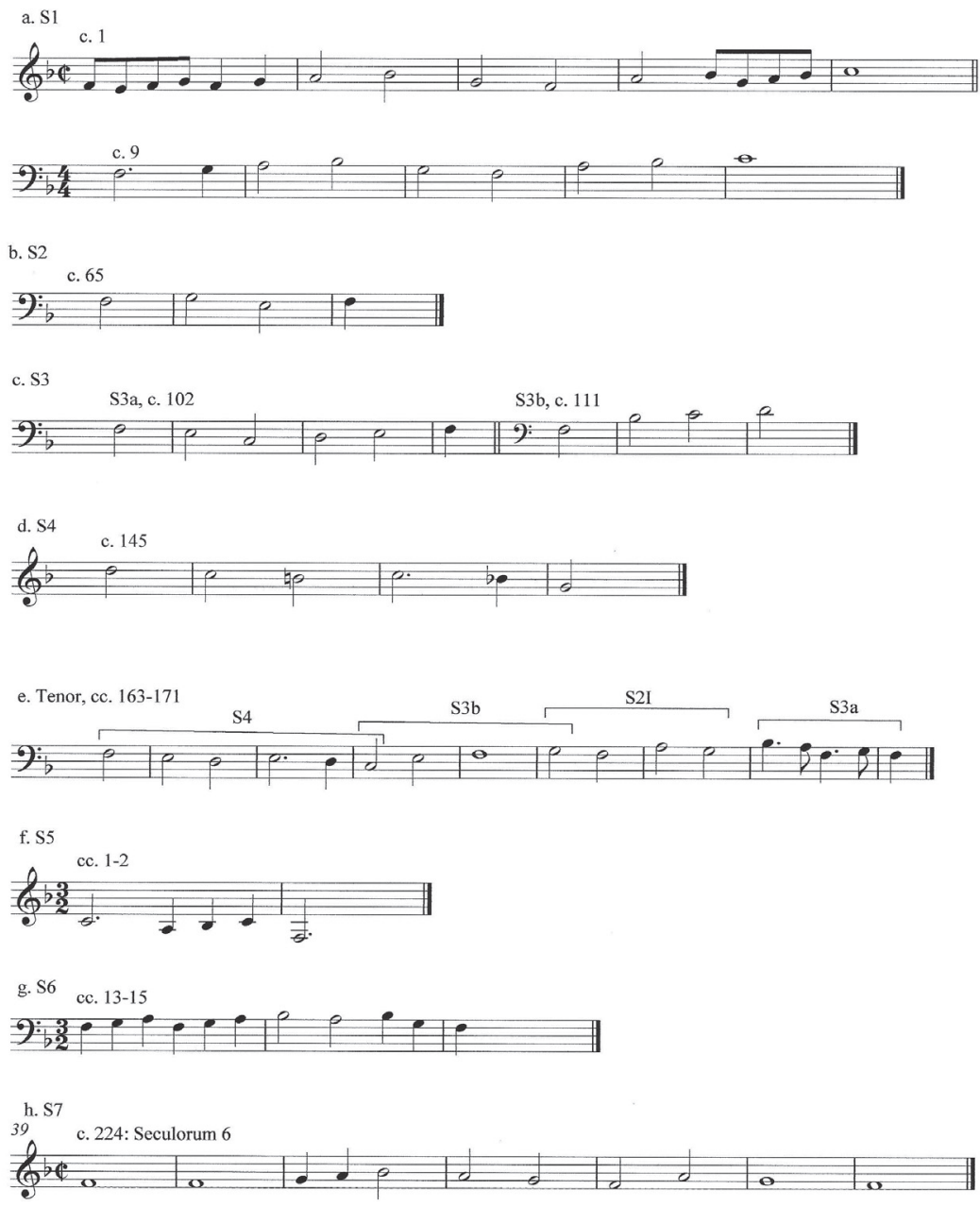

8 Siguiendo la práctica de Tomás de Santa María y otros teóricos espańoles de la época, utilizaremos el término seculorum para referirnos a la entonación salmódica de un modo. Véase SANTA MARÍA, 1565/1972, vol. I: fols. 64r-66r. 
Llama la atención en este extenso tiento no sólo la profusión de sujetos, sino también la variedad de procesos formales utilizados en diversas secciones: variación y fragmentación de la textura en forma de dúos en la sección S1; episodios contrapuntísticos libres en la secciones S2, S3 y sección final, además del libre desarrollo sobre S1 en la segunda parte (cc. 24-42); y progresión canónica en S4.

\section{Coherencia temática.}

Obras 68 se caracteriza por las múltiples relaciones entre sus sujetos, y por la coherencia temática que presentan los siete distintos sujetos. En primer lugar, notemos la presencia de la matriz melódica proporcionada por la entonación salmódica del modo 6 (seculorum 6, abreviado en el ejemplo 2 como sec. 6). S7 está muy directamente derivado del contorno del seculorum 6, como puede verse en el ejem- plo 2h. Tanto S1 como S4 tienen estructuras melódicas cuyo contorno puede derivarse directamente del seculorum 6 . En el ejemplo $2 \mathrm{~g}$ vemos como $\mathrm{S} 1$ y $\mathrm{S} 4$ se combinan para crear S7, y por tanto su estrecha relación con el seculorum 6.

$\mathrm{S} 1$, por su parte, puede dividirse en dos segmentos que generan S3a y S3b respectivamente (ejemplo 2a). Así, S3a es una inversión retrograda (RI) del primer motivo de $\mathrm{S} 1$ (S1RI en el ejemplo 2b), mientras que S3b está derivado del segunto motivo de $\mathrm{S} 1$, con una ligera variación interválica (la tercera ascendente se convierte en una cuarta ascendente, ver ejemplo 2c). S5 presenta el mismo contorno triádico que S1, pero en inversión (ejemplo 2d). Finalmente, S6 no sólo puede leerse como una combinación de $\mathrm{S} 4$ en orden retrógrado (S4R) y S1 (ejemplo 2e), sino que además la retrogradación de S6 (S6R) se identifica con el seculorum 6 y con S7 (ejemplo 2f).

Ejemplo 2: Coherencia temática en Obras 68
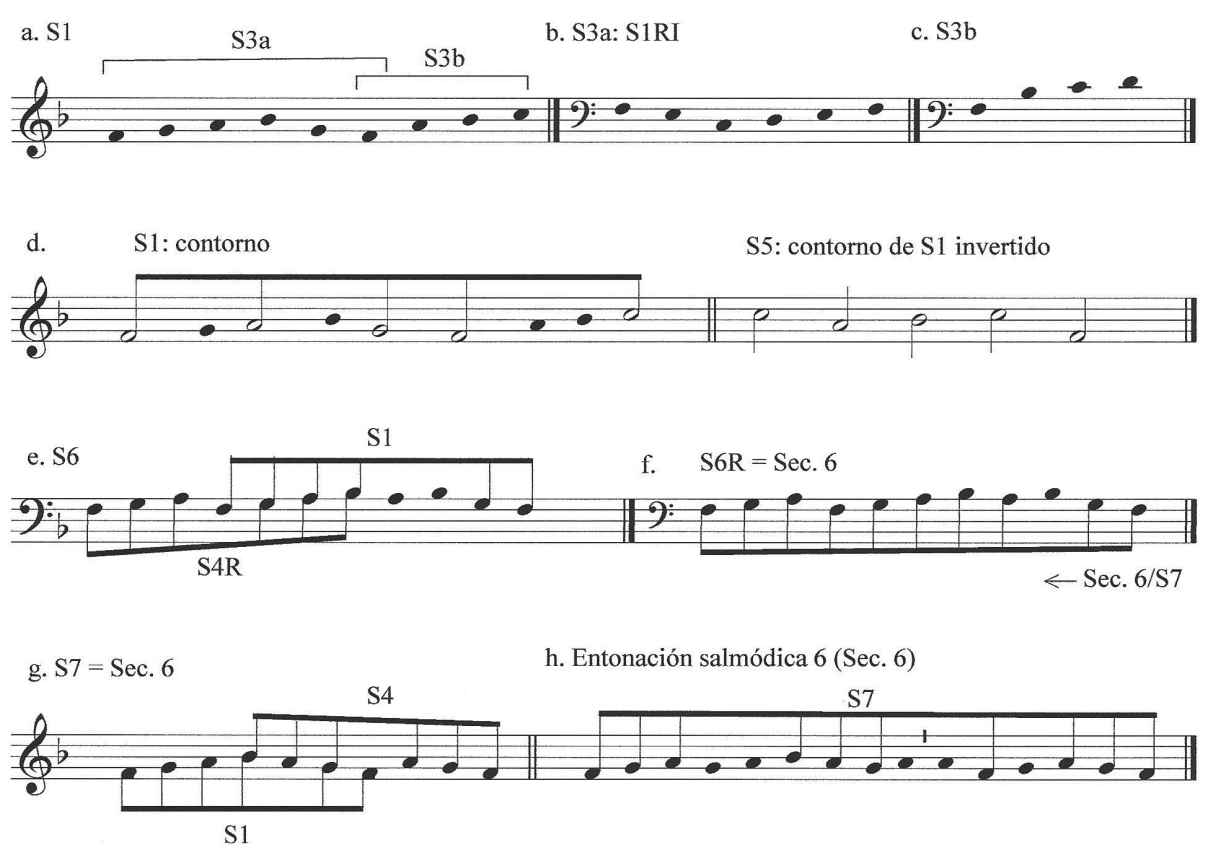


\section{Modalidad.}

Obras 68 es un ejemplo claro de tiento del sexto modo con $\mathrm{Sib}$ en la armadura. El ambitus total de cada una de las voces es muy amplio, abarcando las octavas auténtica y plagal. Pero la tesitura del tiple y el tenor a menudo seńala la octava plagal, Do-Do. El énfasis melódico en la nota La (la repercussa 6) y el intervalo Fa-La (la repercussio 6) es considerable, particularmente en los sujetos S1, S2 (entradas del alto y el tiple, cc. 69-72), S5, S6 y S79. Las cadencias principales de la primera seccion son en $\mathrm{La}$ (cc. 48 y 67), y todas las demás cadencias estructurales (que cierran secciones) del resto del tiento son en Fa (cc. 101 para la sección S2, 145 para la sección S3, 176 para S4 y concluyendo la primera parte; 13 para S5, 24 y 42 para S6, 67 para S7, y 79 como cadencia final). No hay cadencias estructurales en Do en toda la obra.

Las cadencias en La son, sin embargo, "seudo-cadencias". El tenor y bajo en los cc. 47-48 actuan como si la cadencia fuese en La, pero de hecho esta cadencia resuelve a su vez en la cadencia en Re en el compás siguiente (es decir, una "cadencia doble", en la que una cadencia conduce a otra en el compás siguiente). De forma similar, la cadencia "en La" que cierra la seccion S1 (compás 67) es realizada por el tiple y el alto, pero la resolución del bajo a Fa crea una cadencia rota sobre $\mathrm{Fa}$ (o "cláusula hurtada", en términos de Tomás de Santa María), aunque el papel cadencial del La es seńalado por los retardos cadenciales tanto en el compás 47 como en el 66, y por el hecho de que todas las voces en la sección S1 acaban en La (tenor y bajo en el c. 48, y tiple y alto en el 67). Finalmente, la presencia del seculorum 6 en S7 confirma el modo 6 ya indicado por los sujetos y el esquema cadencial.

\section{Conclusiones.}

Obras 68 es el tiento más largo y brillante de Cabezón. Está escrito en un estilo organístico idiomático y virtuoso. La compleja forma seccional de esta obra está unificada por su diseńo tonal y por la cohesión temática. El sujeto $\mathrm{S} 1$ que abre el tiento está directamente derivado del seculorum 6 , que a su vez cierra la obra en forma de S7. El seculorum es también la matriz temática para dos otros sujetos, S4 y S6. S1 por su parte se divide en dos motivos que

9 La repercussa es la nota de recitación (o tenor) en la entonación salmódica, y tiene un papel esencial en la definición de un modo, tanto como nota de inflexión melódica como en su función de nota cadencial interna. La repercussio, por otro lado, es el intervalo estructural de un modo, entre la nota final y la repercussa. generan los sujetos S3a y S3b, además de estar también relacionado con S5 y S6. La forma del tiento se redondea con la vuelta de $\mathrm{S} 1$ en la segunda parte, primero como conexión entre S5 y S6, y después en forma de principal material temático desarrollado en el episodio que precede al seculorum en S7. La recapitulación de cuatro sujetos previos al final de la primera parte seńala aún más la deliberada preocupación por parte de Cabezón con la unidad temática y formal. La variedad, por otro lado, viene proporcionada por figuraciones rítmicas contrastantes, por la diversidad de técnicas de desarrollo y por la sección central en compás triple. Desde el punto de vista modal, la estructura de este tiento es clásica del modo 6, con cadencias en Fa y $\mathrm{La}$, aunque las segundas funcionan como si fuesen en $\mathrm{La}$ en cuanto al contrapunto de las voces cadenciales, pero en realidad una conduce a una cadencia en Re, y otra es una cadencia rota en $\mathrm{Fa}$.

\section{OBRAS DE MÚSICA 65 (TIENTO 8), MODO 8}

\section{Forma .}

Obras 65 está dividido en tres secciones. La primera sección (cc. 1-53) incluye la exposición del sujeto S1 (cc 1-29) y un episodio en contrapunto libre (cc. 29-53). Conectando estas dos subsecciones, el alto presenta de forma prominente una cita de la entonación salmódica del modo 5 en Do (seculorum 5/Do, cc. 24-32). La seccion central (cc. 53-126) abre con la exposición del sujeto S2 (53-74). Un sujeto relacionado se expone en los cc. 74-83 (S2b) y es tratado en stretto en los cc. 83-94. Un desarrollo sobre S1 en los cc. 94-117 cierra la sección, utilizando técnicas como inversión y fragmentación motívica del sujeto. Un puente basado en un giro melódico del seculorum 5 (cc. 117-126) lleva a la sección final.

La sección final incluye la exposición del sujeto S3 (alto, cc. 126-133), con su variante S3b (cc. 135-149) y un pasaje conclusivo (cc. 160-185) basado en una serie de cadencias dobles en Do (es decir, aparentes cadencias en Sol que conducen a cadencias en Do en el compás siguiente; véanse los compases 159, 166 y 174-estas dos últimas son cadencias rotas, además de dobles) que llevan a la cadencia plagal final en Sol. Las técnicas de desarrollo formal utilizadas por Cabezón incluyen episodios en estilo contrapuntístico libre (S1 y sección final), stretto (S2b) y desarrollo temático por medio de inversión y fragmentación motívica. 


\section{Modalidad.}

Obras 65 es uno de los tientos de Cabezón que presenta ambigüedad modal de forma estructural, es decir, que afecta al tiento completo. Este tipo de ambigüedad modal estructural ocurre, por ejemplo, entre los modos 1 y 3 (dórico y frigio), debido a las cadencias comunes en La. Ocurre también entre los modos de los pares tritus (modos 5 y 6 ) y tetrardus (modos 7 y 8 ) respectivamente, debido al Sib en la armadura de las composiciones en los modos 5 y 6 . Como era costumbre entre compositores renacentistas, todas las obras de Cabezón en los modos 5 y 6 llevan un Sib en la armadura de clave. Esto produce un problema obvio de definición modal: la quinta Fa-Sol-La-Sib-Do pertenece a la cuarta especie, y por tanto a los modos tetrardus. El problema fué normalmente reconocido por los teóricos renacentistas. La solución más frecuente, ya adoptada por Tinctoris, era la de aceptar dos posibles especies de octava para los

Ejemplo 3: Relación entre los modos tritus con $\mathrm{Si}$ b y los modos tetrardus

a. La octava del modo 5 (tritus) con Si b

Modos tritus con Si b :

cuarta especie de quinta + tercera especie de cuarta

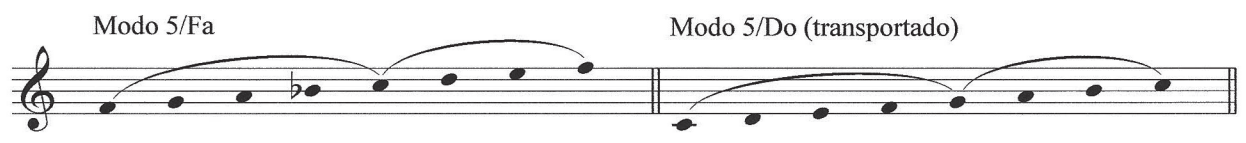

b. Ambigüedad entre tritus y tetrardus

Modo 8/Sol

Octava Re-Sol-Re (nivel plagal) Octava Sol-Re-Sol (nivel auténtico)

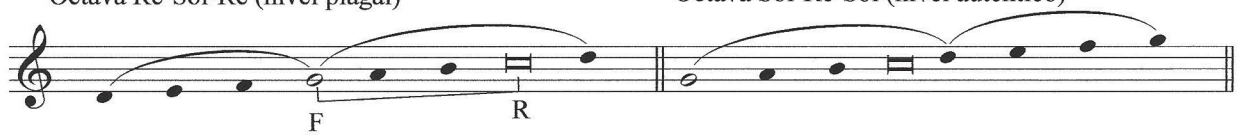

Pero por division de la octava en Do (R8) en el modo 8/Sol:

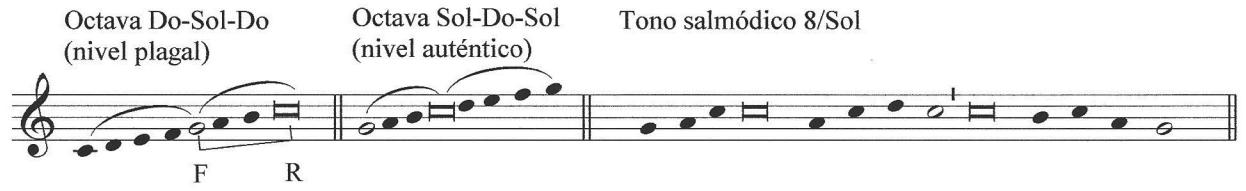

Modo 5/Do

Octava Do-Sol-Do Octava Sol-Do-Sol Tono salmódico 5/Do

(nivel auténtico) (nivel plagal)

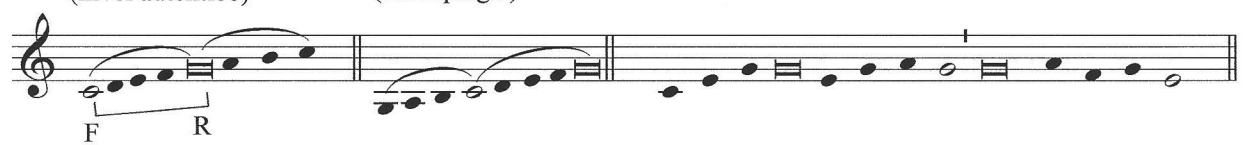


modos tritus, una con $\mathrm{Sib}$ y otra con $\mathrm{Si} \mathfrak{t}^{10}$. La postura de Tomás de Santa María es igualmente pragmática: el teórico dominico declara que la especie de quinta para los modos 5 , 6,7 y 8 es la misma, mientras que la especie de cuarta cambia entre los modos tritus y tetrardus ${ }^{11}$. Otros teóricos, como Vicentino, Bermudo y Aiguino, consideran que los modos tritus con $\mathrm{Si} b$ son modos mezclados: una cuarta especie de quinta (característica de los modos 7 y 8) está mezclada con una tercera especie de cuarta (propia de los modos 5 y 6), como ilustra el ejemplo $3 \mathrm{a}^{12}$. Bermudo, sin embargo, se resiste al empleo del Sib en los modos tritus. Los músicos que tańen los modos 5 y 6 con un Sib, según Bermudo, rompen la octava de estos modos, y violan su estructura esencial. El modo 6 con Sib, dice Bermudo, suena como el modo 8; o más bien, insiste, es el modo 8; o de hecho, concluye, no es ni uno ni otro ${ }^{13}$.

Al margen de las preferencias de Bermudo, el Sib es un hecho generalizado en composiciones en los modos 5 y 6 en el siglo XVI. Y mientras que para Glareano los modos en $\mathrm{Fa}$ con $\mathrm{Sib}$ eran simplemente transposiciones a la cuarta superior de los modos jónico e hipojónico, los seguidores del sistema modal de ocho modos, incluidos Cabezón y Santa María, hubiesen tenido una visión distinta de estos modos: lo normal para los modos 5 y 6 en Fa en un contexto polifónico en el siglo XVI sería llevar un Sib en la armadura. El modo con final en Do y ningún bemol en la armadura sería entonces un modo 5 o 6 tranportado a la cuarta inferior.

La ambigüedad entre los modos tritus y tetrardus, sin embargo, va más allá de su especie de quinta común. Considérense, por ejemplo, los elementos característicos del modo 8 en Sol reproducidos en el ejemplo 3b: la final es Sol, la repercussa $(\mathrm{R} 8)$ es Do, las principales notas cadenciales son Sol y Do, y una frecuente división de la octava es en la repercussa Do (Sol-Do-Sol) en vez de en la cofinal Re (Sol-ReSol). Como resultado, las octavas características del modo

10 "Cualquiera de estos dos modos [5 o 6] puede formarse con el cuarto tipo de diapente, algo que debería hacerse con la menor frecuencia posible", TINCTORIS, 1976: 12. Y también: "Dado que es cierto que el quinto tono y el sexto... se pueden formar con este mismo cuarto tipo de diapente, hay que preguntar qué diferencia hay entre su formación y la formación del séptimo y el octavo. Respondemos que, al contrario, hay entre los primeros y los segundos más diferencias que similitudes. Porque aunque el quinto y el sexto tono estén formados por el cuarto tipo de diapente, el primero de estos requiere el tercer tipo de diatessaron por encima, y el segundo lo requiere por debajo; además del cuarto tipo de diapente con el que también se forman, el séptimo tono requiere el primer tipo de diatessaron por encima y el octavo lo requiere por debajo". Ibid, p.14. Las traducciones del inglés son mías.

11 SANTA MARÍA, 1565/1972: fol. I:62r.

12 VICENTINO, 1555/1959: fol. 51; BERMUDO, 1555/1957: fols. 72v y 82r; AIGUINO, 1581: fols. 76v-77v.

13 BERMUDO, 1555/1957: fols. 72v-73r.
8 en la práctica son Sol-Do-Sol y su complemento plagal, Do-Sol-Do. Veamos ahora el modo en Do, que resulta de transportar la octava del modo 5, Fa-Fa con Sib, a la cuarta inferior. La escala original en Fa con $\mathrm{Sib}$ se convierte, por la transposición, en una escala en Do sin alteraciones. La final es Do, la repercussa es Sol, y la octava característica es Do-Sol-Do. Siguiendo la práctica de Santa María de aceptar el cuarto grado bemolizado ( $\mathrm{Sib}$ si la final es $\mathrm{Fa}$ ) como un hecho en los modos tritus, consideraremos la octava Do-SolDo como propia del modo 5 transportado, y la octava SolDo-Sol como propia del modo 6 transportado. Notemos que las notas estructurales de estos modos tritus (Do y Sol), así como sus octavas, son las mismas que las del modo 8 en Sol (tetrardus) que hemos examinado anteriormente. Como paralelo final entre los modos 8 en Sol y 5 en Do, consideremos sus respectivas entonaciones salmódicas. Dado que el tenor (repercussa) y la mediatio (cadencia media) del tono salmódico 8 en Sol están situados una cuarta por encima del tenor y mediatio del tono salmódico 5 en Do, las dos entonaciones pueden ser utilizadas con una relación de sujeto y respuesta en un contexto imitativo.

En resumen, las notas estructurales y cadenciales de estos dos modos son las mismas, aunque con funciones intercambiadas : en el modo 8 en Sol la final es Sol y la repercussa es Do, mientras que en el modo 5 en Do la final es Do y la repercussa Sol. Las octavas características de ambos modos son las mismas, y sus entonaciones salmódicas tienen contornos melódicos similares a distancia de cuarta. Una diferencia esencial entre los dos modos, sin embargo, es que el intervalo estructural del modo 5 en Do es una quinta, Do-Sol, mientras que el intervalo estructural del modo 8 en Sol es una cuarta, Sol-Do. Es decir, la relación interválica que distingue estos dos modos es la misma que distingue los modos frigio (cuarta Mi-La) y eolio (quinta La-Mi).

El tiento Obras 65 está idenfiticado como del modo 8, con final en Sol, en la edición original de las Obras. Los signos inequívocos que apuntan al modo 8 en este tiento son las cadencias en Sol y Do, el esquema de notas iniciales de las cuatro voces en las entradadas imitativas de S1 y S2 (Sol-Do-Sol-Do y Do-Sol-Do-Sol respectivamente), el énfasis melódico en la repercussio 8 (la cuarta Sol-Do), particularmente en S1, S2 y S2b, y la cadencia plagal final en Sol, precedida por una serie de cadencias en Do (una cadencia auténtica en el c. 160 y dos cadencias rotas en los cc. 167 y 175). Las especies interválicas del modo 8 están presentes a dos niveles tonales, Sol y Do, como puede verse en la imitación inicial: el alto y bajo seńalan la quinta Do-Sol, como parte de la octava Do-Sol-Do, mientras que el tiple y el tenor marcan la quinta Sol-Re. La ambigüedad con el modo 5/Do está por tanto creada por las cadencias comunes entre los 
modos 5/Do y 8/Sol, y por el doble nivel de las especies interválicas: la octava Sol-Re-Sol es propia del modo 8/Sol, mientras que la octava Do-Sol-Do es característica de ambos modos.

\section{Coherencia Temática.}

La ambigüedad es acentuada y confirmada por el material temático del tiento. Al igual que Obras 68, Obras 65 representa también un caso de cohesión temática entre sus sujetos, como podemos comprobar en el ejemplo 4. Vemos en primer lugar en este ejemplo que todos los sujetos están directamente relacionados con las entonaciones salmódicas 8/Sol y 5/Do. He seńalado cuatro motivos melódicos en los tonos salmódicos (a, b, c, d) que, como veremos inmediatamente, generan el material temático completo del tiento (ejemplo 4a). El sujeto inicial en el alto, S1, tiene un contorno derivado del tono salmódico 5/Do, mientras que la respuesta del tiple puede referirse claramente al tono salmódico 8/Sol, ilustrando así la relación de sujeto/respuesta entre estos dos tonos salmódicos, como hemos seńalado anteriormente (ejemplo 4b). Tanto el sujeto S1 como la respuesta incluyen una nota repetida, seguida por el motivo a, con su característico salto de tercera, y por el motivo b, una cuarta descendente. La versión de S1 presentada por el alto en los cc. 24-32, una clara referencia al tono salmódico 5/ Do dentro de este tiento en el modo 8/Sol (ejemplo 4c), apunta de nuevo a la ambigüedad estructural entre estos dos modos y estos dos tonos salmódicos, y demuestra incluso más claramente la relación entre $\mathrm{S} 1$ y el seculorum 5/Do.

En el ejemplo 4d vemos que S2 combina retrogradaciones de los motivos c (en forma de cR) y b (en forma de bR), mientras que $\mathrm{S} 2 \mathrm{~b}$ resulta de la combinación de $\mathrm{S} 2$ (motivo c, retrogradado como cR) y S1 (motivo a', inver- tido como a'I). El puente en los cc. 118-127 está basado en citas literales del motivo d, la terminatio del tono salmódico 5/Do. S3 (ejemplo 4e) está derivado directamente de S1: la cuarta descendiente que cierra S1 (motivo b) se convierte en la cuarta ascendente que abre S3 (b invertido como bI), seguida por los motivos a y a' traslapados, el primero retrogradado $(\mathrm{aR})$ y el segundo invertido (a'I). La relación entre S3b y S1 es incluso más directa (ver ejemplo 4e): S3b abre con una nota repetida, seguida por una cuarta descendiente (motivo b) superpuesta con el motivo a. Esta conexión entre S1 y S3, junto con la breve vuelta de S1 en los cc. 149-154 (tiple y alto), proporciona al tiento un sentido de simetría formal que refuerza todavía más su unidad arquitectónica.

\section{Conclusiones.}

Obras 65 es sin duda una de las obras maestras de Cabezón. Este tiento relativamente breve no contiene pasajes de virtuosismo ni secciones contrastantes. Su característica más notable es su gran unidad estilística y compositiva, definida por su regularidad rítmica, su coherencia tonal, su simetría formal y, sobre todo, el hecho de que todo su material temático puede derivarse, aunque no siempre de forma obvia, de la entonación salmódica 8. El papel del seculorum es por tanto central en este tiento, no sólo como un elemento de confirmación modal, sino también como matriz temática. El factor de ambivalencia dentro de esta construcción tan sólidamente unificada viene dado por la amigüedad estructural entre los modos 8 y 5 . Y, paradójicamente, esta ambigüedad está acentuada por el mismo elemento que contribuye a la unidad del tiento: el seculorum 8, debido a su similitud con el seculorum 5. 
Ejemplo 4: Coherencia temática en Obras 65
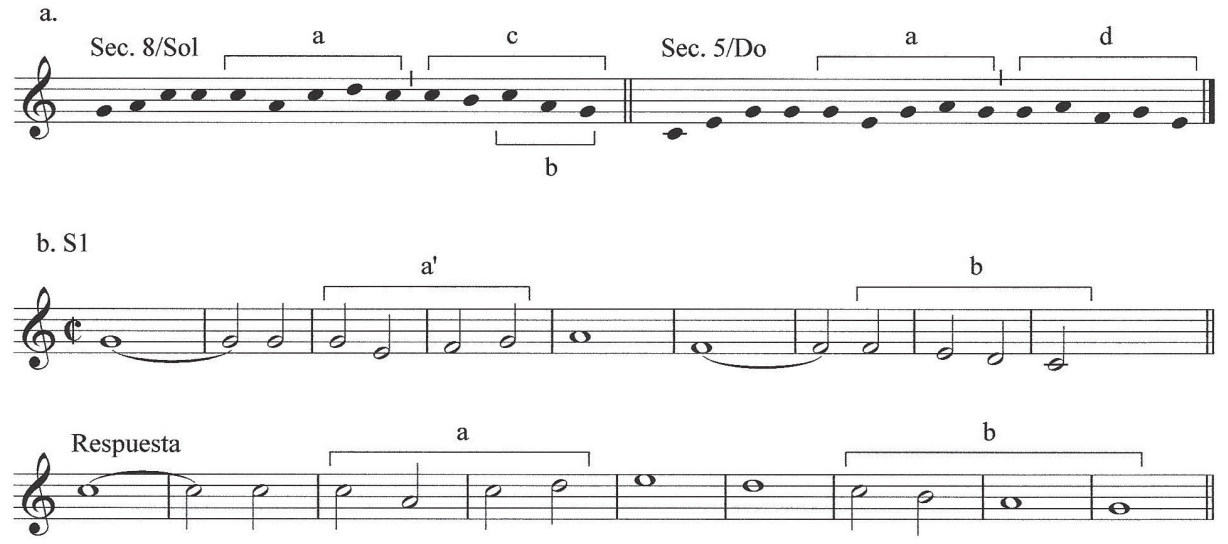

c. Alto, cc. 24-32: Sec. 5/Do
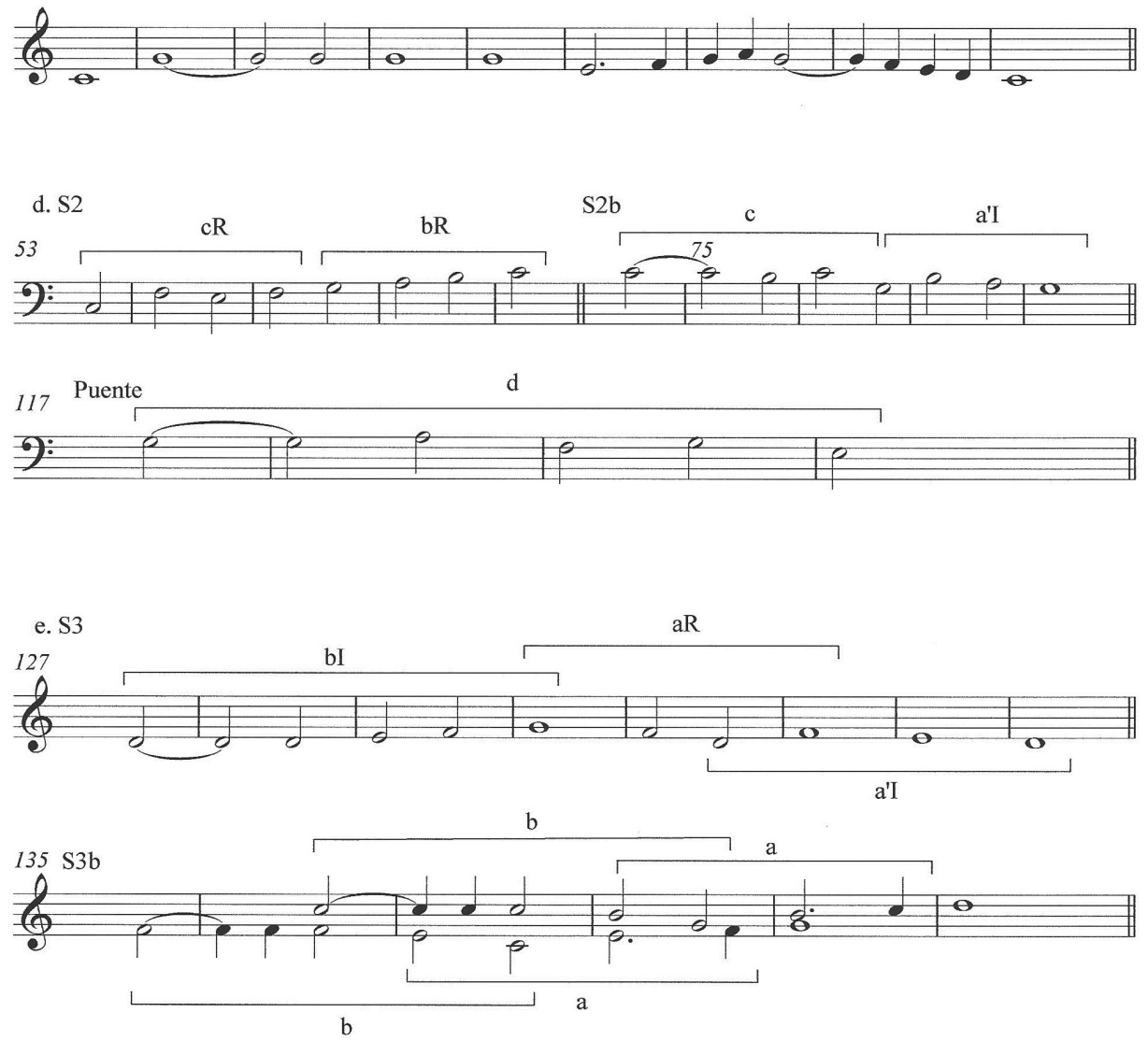


\section{OBRAS DE MÚSICA 67 (TIENTO 10), MODO 1}

Obras 67 es uno de los tientos más breves de Cabezón (59 compases), y también quizá el caso más interesante de cambio modal en toda la producción del maestro burgalés. Se trata de un tiento de carácter recogido y contemplativo, de gran belleza sin ningún tipo de concesiones al virtuosismo o al despliegue técnico al teclado, una verdadera obra maestra de economía compositiva, estructura tonal, y concentrada riqueza de contenido musical. El tiento está compuesto en el modo 1, según indica la edición original de las Obras. Recordemos que las cadencias principales del modo 1 son en Re, la final del modo, y en La, la repercussa. Dado que la parte central del tiento, como veremos inmediatamente, está en el modo 3, repasemos las características de este modo.
Según el Arte de tańer fantasía (fol. I:68r), las notas cadenciales para el modo 3 son Mi (la final del modo), Do (la repercussa) y Sol para las cadencias de paso. El modo 4, por otro lado tiene sus cadencias en Mi y La (la repercussa 4). Aunque Santa María no menciona las frecuentes cadencias en La en el modo 3 (aparte de mencionar que La es la principal cadencia en el seculorum del modo 3), su ejemplo de fantasía en el modo 3, la fantasía 8 (fol. I:68r) incluye cadencias en $\mathrm{Mi}$, La, Do, y Sol, al igual que las cadencias que encontramos en el único tiento de Cabezón en el modo 3, Obras 63 (tiento 6). El sujeto inicial en la fantasía 8 de Santa María, reproducido en el ejemplo 5, es también característico del modo 3: Mi-Sol-La-Si-Do, un tema que subraya la repercussio 3, la sexta menor Mi-Do.

Ejemplo 5: Tomás de Santa María, Fantasía 8, sujeto inicial

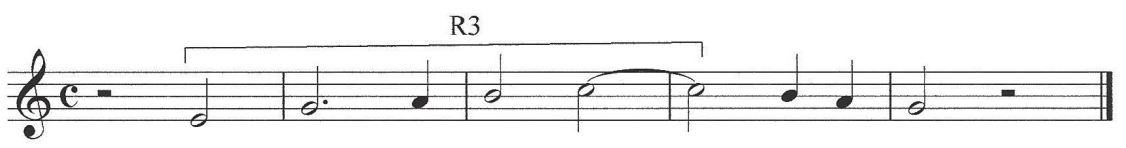

Volviendo ahora a Obras 67, vemos que la primera sección (cc. 1-26) está en el modo 1, con cadencias en Re (c. 15) y La (c. 26). La sección intermedia (cc. 26-43, reproducida en el ejemplo 6), por otro lado, está en el modo 3. Las especies interválicas prominentes en esta sección son la segunda especie de cuarta (Mi-La, bajo, cc. 37-42), la repercussio 3 (R3, Mi-Do, tiple y tenor, cc. 26-30, y tiple, cc. 32-34), la octava Mi-Mi dividida en La (Mi-La-Mi, tenor, cc. 28-43), y la octava La-La dividida en Mi (La-Mi-La, bajo, cc. 31-42). Todas estas especies son características del modo 3. Las cadencias de esta sección son en La (cc. 36 y 43) y Do (rota, c. 30). El sujeto de esta sección intermedia, S2, es el característico sujeto del modo 3 que acabamos de ver en la fantasía 8 de Santa María, ahora presentado como Mi-Sol-La-Si-Sol-Do en el tiple, y como Mi-Sol-La-Do en el tenor. 
Ejemplo 6: Cabezón, Obras 67, sección intermedia
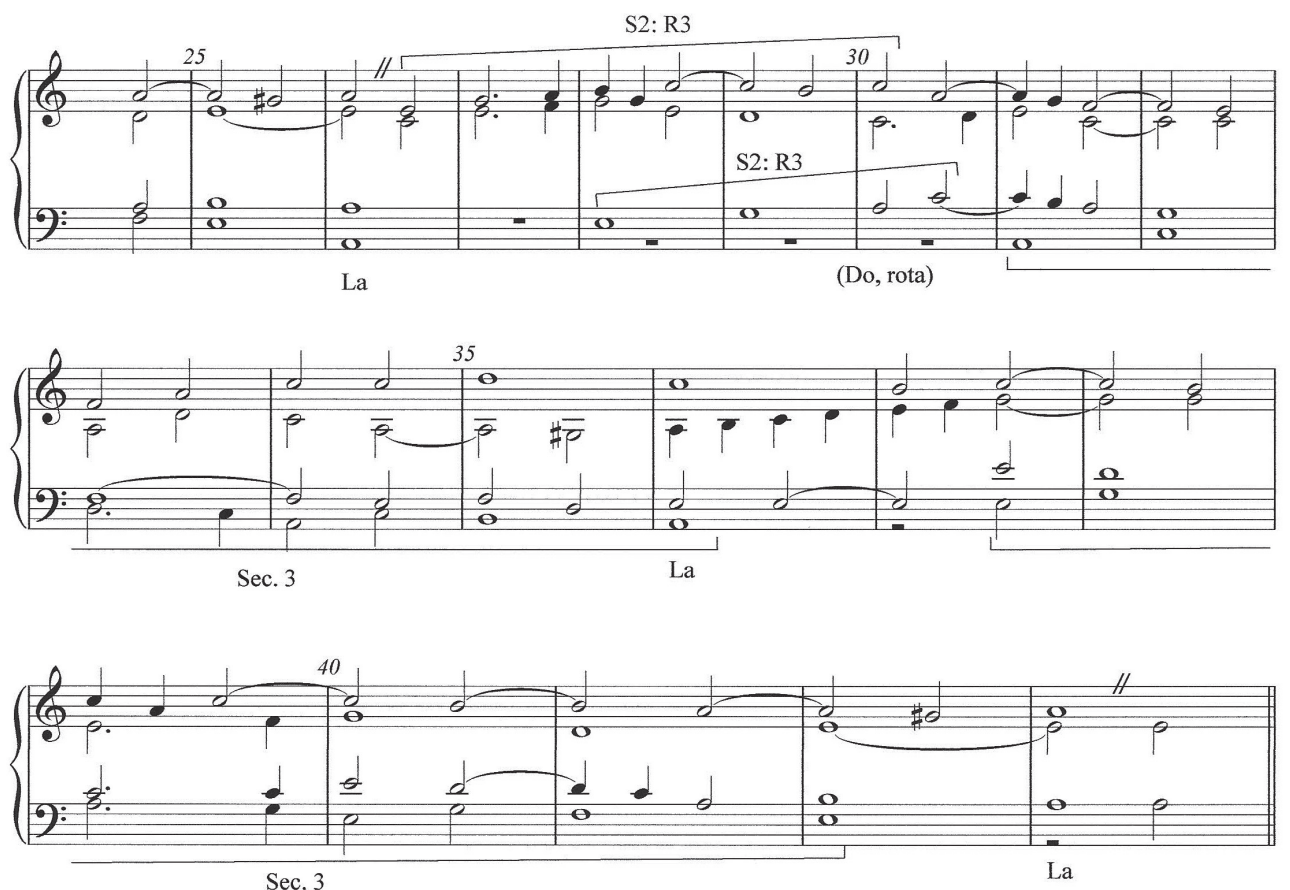

La frase del bajo en esta sección resulta ser una versión simplificada del seculorum 3, expuesta dos veces en los niveles tonales de La y Mi respectivamente (ver el ejemplo 7 para una comparación del seculorum 3 con la frase del bajo en esta sección; recordemos que La es la principal nota cadencial de la entonación salmódica 3). La sección final del tiento (cc. 43-59) constituye una vuelta al sujeto original, $\mathrm{S} 1$, que es presentado en primer lugar en forma de especies melódicas de los modos 3 y 1 simultáneas: las cuartas Mi-La en el bajo y La-Re en el tenor, cc. 44-48. Después de una cadencia en La en el c. 51, la sección concluye con S1 claramente en modo 1 (cc. 54-58) y una cadencia final en Re.

Ejemplo 7: Cabezón, Obras 67, frase del bajo, cc. 31-36

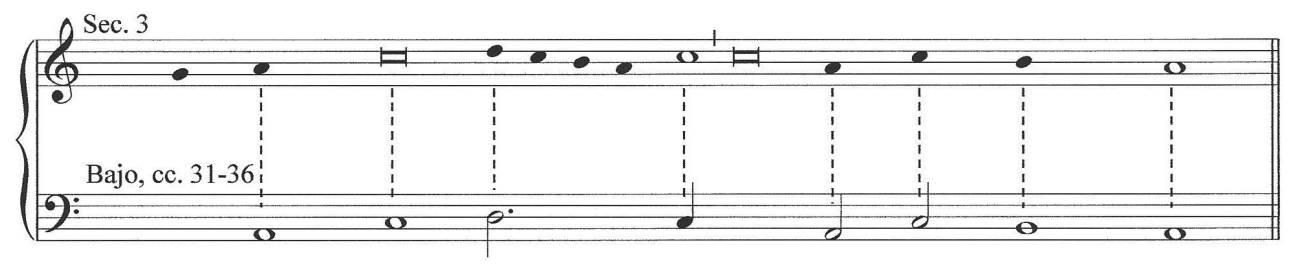

La mezcla modal entre los modos 1 y 3 , que como indica Bernhard Meier ya fué descrita por Erasmo y Gafori como A Dorio ad Phrygium, es una de las más comunes e interesantes, a pesar de la gran diferencia entres las escalas dórica y frigia. ${ }^{14}$ El nexo o elemento común entre los dos modos es la nota La, que ocupa una posición esencial en

14 MEIER, 1956. 
ambos modos. La nota La, situada centralmente una quinta por debajo de Re y una quinta por encima de Mi, es la repercussa y cofinal del modo 1 , la repercussa del modo 4, y la principal differentia o nota cadencial de la entonación salmódica 3. La octava La-Mi-La es otro elemento esencial común entre los pares de modos dóricos y frigios. Debido a las imitaciones a la quinta superior, la octava Re-La-Re, que define el modo 1, es normalmente imitada por la octava LaMi-La, que es por tanto también propia del modo 1. Por otro lado, ya hemos visto que las cadencias en La crean una división de la octava del modo 3 en la nota La, en forma de MiLa- Mi, o, en voces con un ambitus plagal, La-Mi-La. Esta doble función de la nota La y la octava La-Mi-La (esenciales en los pares de modos protus y deuterus) fué ampliamente utilizada por los compositores renacentistas para establecer la conexión entre los modos 1 y 3 , en apariencia tan contrastantes. Cabezón de hecho se basa en esta relación para crear pasajes de transición entre las secciones de Obras 67. Así, la sección S2 es precedida por un pasaje de ambigüedad entre los modos 1 y 3 que prepara la confirmación del modo 3: la nota Do (repercussa 3) está claramente seńalada en el tiple (cc. 20-22), y la primera especie de quinta sobre La (La-Mi, tenor, cc. 23-26) aparece simultaneamente a la octava LaMi-La en el bajo. El fragmento cierra con una cadencia en La, común a ambos modos. Hay que notar que la imitación inicial sobre el sujeto $\mathrm{S} 1$ ya anuncia los principales elementos de ambigüedad en los que se basa el tiento: S1 se mueve dentro de la cuarta descendiente $\mathrm{La}-\mathrm{Mi}$, y la respuesta es la cuarta descendiente Mi-Si; el esquema imitativo de las cuatro voces es La-Mi-La-Mi. Aunque todo ello es posible en el modo 1 , los intervalos presentados melódicamente y en la imitación en este principio del tiento son más propios de un modo frigio que de uno dórico.

En resumen, Cabezón realiza el cambio de modo por medio de un pasaje en el que los elementos comunes funcionan como conexión entre los dos modos, y nos informa que la nueva sección está inequívocamente en el modo 3 por medio de un característico sujeto de este modo, y derivando la frase del bajo del seculorum 3. La maestría de Cabezón queda patente no sólo en su forma de introducir la mezcla modal, sino también en su hábil conexión entre los procesos formales y tonales de la obra. El tiento se divide en tres partes, de las cuales la parte central representa una excursión tonal que nos aleja del modo y sujeto principales, mientras que la tercera sección funciona como una vuelta tanto al modo como al sujeto originales, en un diseńo ternario perfectamente simétrico y equilibrado.

\section{BIBLIOGRAFÍA}

AIGUINO, Illuminato, Il tesoro illuminato de tutti i tuoni di canto figurato, Venecia, G. Varisco, 1581.

BERMUDO, Juan, Declaración de instrumentos musicales, Osuna, Juan de León, 1555; ed. facsímile, Documenta musicologica, serie 1, no. 11, Kassel, Bärenreiter, 1957.

CABEZÓN, Antonio de, Obras de música para tecla, arpa $y$ vihuela, Felipe PEDRELL (ed.), revisada H. ANGLÉS, Monumentos de la Música Española XVIIXIX, Barcelona, Instituto Español de Musicología, 1966.

CABEZÓN, Antonio de, Obras de música para tecla, arpa $y$ vihuela, Javier ARTIGAS PINA et al. (eds.), Zaragoza, Consejo Superior de Investigaciones Científicas/ Institución "Fernando el Católico", 2010.

JACOBS, Charles (ed.), The Collected Works of Antonio de Cabezón, Brooklyn, Institute of Medieval Music, 1967-86.

KASTNER, Macario Santiago (ed.), Antonio de Cabezón, Tientos und Fuguen aus den "Obras", Maguncia, Schott, 1958.

MEIER, Bernhard, "The musica reservata of Adrianus Petit Coclico and its Relationship to Josquin", Musica Disciplina, 10 (1956): 69-79.

ROIG-FRANCOLÍ, Miguel Ángel, Compositional Theory and Practice in Mid-Sixteenth-Century Spanish Instrumental Music: The "Arte de tañer fantasía" by Tomás de Santa María and the Music of Antonio de Cabezón, Tesis doctoral, Universidad de Indiana, 1990.

ROIG-FRANCOLÍ, Miguel Ángel, "En torno a la figura y la obra de Tomás de Santa María: aclaraciones, evaluaciones y relación con la música de Cabezón", Revista de Musicología, 15 (1992): 55-85.

ROIG-FRANCOLÍ, Miguel Ángel, "Modal Paradigms in Mid-Sixteenth Century Spanish Instrumental Composition: Theory and Practice in Antonio de Cabezón and Tomás de Santa María”, Journal of Music Theory, 38 (1994): 249-291.

ROIG-FRANCOLÍ, Miguel Ángel, "Dos tientos de Cabezón basados en tonos de Magnificat", Revista de Musicología, 21 (1998): 1-19.

ROIG-FRANCOLÍ, Miguel Ángel, "Paradigms and Contrast in Sixteenth-Century Modal Structure: Commixture in the tientos of Antonio de Cabezón", Journal of Musicological Research, 19 (2000): 1-47. 